\title{
REPRODUÇÃO E LONGEVIDADE DE Orius insidiosus (SAY) \\ (HEMIPTERA: ANTHOCORIDAE) EM DIFERENTES \\ TEMPERATURAS
}

\author{
Simone M. Mendes ${ }^{1}$ \\ Vanda H. P. Bueno' \\ Lívia M. Carvalho'
}

\section{RESUMO}

O conhecimento da temperatura adequada ao desenvolvimento e reprodução é essencial para prever o estabelecimento e o sucesso da utilização de um agente em programas de controle biológico, assim como para a sua criação massal em laboratório. O objetivo deste trabalho foi avaliar a reprodução e longevidade de Orius insidiosus (Say) (Hemiptera: Anthocoridae) em câmaras climatizadas nas temperaturas de 16, 19, 22, 25, 28 e $31 \pm 1{ }^{\circ} \mathrm{C}$; fotofase de 12 horas e UR de $70 \pm 10 \%$. Foram utilizadas inflorescências de picão-preto (Bidens pilosa L.) como substrato de oviposição, e como alimento, ovos de Anagasta kuehniella (Zeller) (Lepidoptera: Pyralidae). Os períodos de pré-oviposição e oviposição de $O$. insidiosus variaram com a temperatura. As porcentagens de fêmeas que ovipositaram foram de $60 ; 81,8 ; 92,8 ; 100 ; 93,3$ e $73,6 \%$ nas temperaturas de $16,19,22,25,28$ e $31^{\circ} \mathrm{C}$, respectivamente. O número de ovos/fêmea foi de 35,$4 ; 169,6 ; 183,0 ; 206,7 ; 142,6$ e 109,4 nessas respectivas temperaturas. A longevidade dos adultos foi influenciada pela temperatura. A curva de oviposição de $O$. insidiosus mostrou que a $25^{\circ} \mathrm{C}$, as fềmeas alcançaram o pico de oviposição no $11^{\circ}$ dia e que $80 \%$ dos ovos foram colocados até o

\footnotetext{
${ }^{1}$ Universidade Federal de Lavras, Departamento de Entomologia, Caixa Postal 37, CEP 37200-000 Lavras, MG.
} 
$30^{\circ}$ dia após a emergência das mesmas. A temperatura de $25^{\circ} \mathrm{C}$ foi a mais adequada para ser utilizada em criações massais de $O$. insidiosus, por maximizar as características reprodutivas das fêmeas desse predador.

Palavras-chave: controle biológico, fecundidade, oviposição, Orius.

\section{ABSTRACT}

The understanding of a suitable temperature for development and reproduction is essential to predict the establishment and the success of a biocontrol agent in biological control programs, as well as for its mass rearing under laboratory conditions. The objective of this work was to evaluate the effect of temperature on reproduction and longevity of Orius insidiosus (Say) (Hemiptera: Anthocoridae). The tests were carried out in climatic chambers at $16,19,22,25,28$ and $31 \pm 1^{\circ} \mathrm{C}$, photophase $12 \mathrm{~h}$ and RH $70 \pm 10 \%$. The farmer's friend inflorescence (Bidens pilosa L.) were used as oviposition substrate, and the eggs of Anagasta kuehniella (Zeller) (Lepidoptera: Pyralidae) as food. The pre-oviposition and oviposition periods and fecundity of $O$. insidiosus were affected by the temperature. The percentage of the females laying eggs were $60,81.8,92.8,100,93.3$ and $73.68 \%$ at $16,19,22,25,28$ and $31^{\circ} \mathrm{C}$ respectively. The total number of eggs/female were 35.4, 169.6, 183.0, 206.7, 142.6 and 109.4 eggs, respectively at the same temperatures. The longevity was different for male and female at different temperatures. The oviposition curve of $O$. insidiosus showed that, under $25^{\circ} \mathrm{C}$, the females reached an oviposition peak on the eleventh day and $80 \%$ of their eggs were laid until the thirtieth day of her lifespan. The temperature $25^{\circ} \mathrm{C}$ is more suitable for the mass rearing of the predator $O$. insidiosus, maximizing its reproductive features.

Key words: biological control, fecundity, oviposition, Orius. 


\section{INTRODUÇÃO}

O conhecimento das adaptações climáticas dos insetos tem papel essencial no manejo integrado de pragas, por prever o tempo de desenvolvimento, taxas reprodutivas e de migração. A temperatura é o fator abiótico mais conhecido que regula o desenvolvimento dos insetos-praga e de seus inimigos naturais (Campbel et al., 1974; Roy et al., 2002).

No controle biológico, o conhecimento da temperatura adequada ao desenvolvimento e reprodução é fundamental para estimativas do sucesso na utilização do agente de controle, seja por métodos de introdução, conservação ou quanto à sua criação massal (Roy et al., 2002). Isso ajuda a selecionar inimigos naturais adaptados às mesmas condições ambientais daquelas das pragas, o que favorece o controle das mesmas. O desconhecimento desse aspecto foi, entre outros, razão de fracasso em muitos programas de controle biológico (Campbel et al., 1974; Roy et al., 2002).

A temperatura e a alimentação têm grande influência na reprodução e desenvolvimento de predadores do gênero Orius Wolff, o que aumenta a velocidade de crescimento de suas populações (Malais \& Ravensberg, 2003). O percevejo predador Orius insidiosus (Say) (Hemiptera: Anthocoridae), em regiões temperadas, apresentam diapausa reprodutiva induzida por condições de fotoperíodo curto (van den Meiracker, 1994). Mas a temperatura pode ter um efeito modificador substancial na resposta ao fotoperíodo (Ruberson et al., 2000).

$\mathrm{O}$ efeito de fatores como fotoperíodo, nutrição e disponibilidade de alimento na performance reprodutiva de $O$. insidiosus coletados na região Sudeste do Brasil, é conhecido (Argolo et al., 2002, Mendes et al., 2002; Mendes et al., 2003). No entanto, poucas são as informações disponíveis sobre o efeito da temperatura em espécies desse gênero presentes em regiões tropicais. Além disso, é sabido que a maximização da eficácia de 
um agente de controle biológico no campo ou em casas de vegetação se dá pela utilização de condições adequadas na sua criação e emprego.

O objetivo desse trabalho foi avaliar a reprodução e a longevidade de $O$. insidiosus em diferentes temperaturas, alimentado com ovos de Anagasta kuehniella (Zeller) (Lepidoptera: Pyralidae), visando à sua criação massal e utilização como agente de controle biológico.

\section{MATERIAL E MÉTODOS}

O experimento foi conduzido no Laboratório de Controle Biológico do Departamento de Entomologia da Universidade Federal de Lavras (UFLA). A criação do predador $O$. insidiosus foi mantida em laboratório, de acordo com metodologia proposta por Bueno (2000) e Mendes \& Bueno (2001), sendo utilizada no experimento a terceira geração. Efeito da temperatura na reprodução e longevidade de $\boldsymbol{O}$. insidiosus. O estudo foi conduzido em câmaras climatizadas com $70 \pm 10 \%$ de UR, fotofase de 12 horas nas temperaturas de $16,19,22,25,28$ e $31 \pm 1^{\circ} \mathrm{C}$.

Ovos com idade inferior a 24 horas foram retirados da criação de manutenção, e após a eclosão das ninfas, essas foram criadas, isoladamente, em placas de Petri (5,0 cm de diâmetro), contendo ovos de $A$. kuehniella, ad libitum, nas temperaturas avaliadas. Após a emergência, os adultos foram sexados (por meio da análise da genitália) e separados em casais na densidade de um casal por placa. Os machos foram mantidos com as fêmeas até o início da oviposição e, depois, as mesmas foram acasaladas a cada sete dias (Mendes et al., 2003).

Os adultos foram isolados em placas de Petri $(5,0 \mathrm{~cm}$ de diâmetro), contendo um pedaço de algodão umedecido $\left(0,5 \mathrm{~cm}^{2}\right)$ com água destilada $\mathrm{e}$ ovos de $A$. kuehniella, ad libitum, como fonte de alimento. Inflorescências de picão-preto (Bidens pilosa L.) foram utilizadas como substrato para oviposição. Foram cortadas as pontas dessas inflorescências, para extração 
do pólen. O substrato de oviposição, os ovos de A. kuehniella e a fonte de água foram trocados diariamente.

Com o auxílio de microscópio estereoscópico avaliou-se os períodos de pré-oviposição e oviposição, o número médio diário e total de ovos/fêmea, bem como a longevidade dos adultos de $O$. insidiosus nas diferentes temperaturas.

Análise dos dados. $O$ experimento foi conduzido em delineamento inteiramente casualisado, com número diferente de repetições por tratamento. Foi realizada análise de variância e também feito o ajuste de equações de regressão em função da temperatura para todos os parâmetros reprodutivos avaliados.

Para a curva de oviposição de $O$. insidiosus foi considerada a temperatura de $25^{\circ} \mathrm{C}$. Foi calculada a média do número de ovos colocados por dia dessa temperatura e observado o número total médio sendo então, obtida a percentagem diária de oviposição. Para a curva acumulada seguiuse o mesmo procedimento apenas considerando a percentagem acumulada do número de ovos/dia e selecionando-se o dia em que $80 \%$ dos ovos foram colocados, segundo metodologia adaptada de Tommasini, 2003.

\section{RESULTADOS E DISCUSSÃO}

O período de pré-oviposição de $O$. insidiosus foi diretamente influenciado pela temperatura (Figura 1A), apresentando uma diminuição desse período com o aumento da temperatura, e sendo cerca de cinco vezes maior na temperatura de $16^{\circ} \mathrm{C}(20,6$ dias $)$ do que em temperaturas acima de $22^{\circ} \mathrm{C}$ (5,2 dias). $\mathrm{O}$ ajuste verificado para este período em $O$. insidiosus foi semelhante ao encontrado para Orius laevigatus (Fieber) (Hemiptera: Anthocoridae) em regiões temperadas, o qual apresentou maior período de pré-oviposição em temperaturas mais baixas (Alauzet et al., 1994). Também, Tommasini \& Benuzzi (1996) observaram que O. laevigatus 
submetido a temperatura de $14^{\circ} \mathrm{C}$ demorou 23 dias para iniciar sua oviposição e, quando em temperaturas mais altas (acima de $17^{\circ} \mathrm{C}$ ), esse período diminuiu gradativamente. O mesmo foi verificado quando fêmeas de $O$. insidiosus foram expostas a temperaturas variando de 18 a $30^{\circ} \mathrm{C}$ (Van Den Meiracker, 1999),

Isso pode indicar que, havendo disponibilidade de alimento, substrato de oviposição e alimento adequado, a temperatura é o fator que exerce maior influência sobre esse período em indivíduos coletados em regiões tropicais, uma vez que de acordo com Argolo et al. (2002), o efeito do fotoperíodo induz a diferenças menores no periodo de pré-oviposição desse predador do que as observadas neste estudo causadas pela temperatura. Á temperatura de $16^{\circ} \mathrm{C}$ fêmeas de $O$. insidiosus apresentaram um maior período de pré-oviposição (20,6 dias) (Figura 1A) e foi observada uma menor porcentagem de fềmeas que ovipositaram (60,0\%) (Tabela 1). De acordo com Chyzik et al. (1995), Orius spp. é mais sensível a baixas temperaturas, independentemente do fotoperíodo. Já Van Den Meiraker (1994) considerou fêmeas de $O$. insidiosus em diapausa reprodutiva aquelas que não ovipositaram sob a ação de fotoperíodos curtos e Ruberson et al. (2000), aquelas cujo período de pré-oviposição foi maior do que 14 dias.

O presente estudo revelou uma sensibilidade maior desse predador quanto a iniciar a oviposição na temperatura de $16^{\circ} \mathrm{C}$ e fotofase de 12 horas. No entanto, como Argolo et al. (2002) e Silveira \& Bueno (2003) não observaram efeitos da variação do fotoperíodo, sob temperatura de $25^{\circ} \mathrm{C}$ na indução de diapausa em indivíduos de $O$. insidiosus presentes em áreas tropicais, é notório que estudos mais conclusivos são necessários com relação à questão de diapausa reprodutiva desse predador coletado nessas regiões.

O período de oviposição de $O$. insidiosus foi influenciado pelas diferentes temperaturas avaliadas, ajustando-se a uma curva de $3^{\circ}$ grau 
(Figura 1B). Nas temperaturas extremas $\left(16\right.$ e $\left.31^{\circ} \mathrm{C}\right)$, a duração desse período foi menor, com 16,9 e 19,8 dias, respectivamente, e maior nas temperaturas de 19,22 e $25^{\circ} \mathrm{C}$. Este resultado mostra que, provavelmente, temperaturas abaixo de $16^{\circ} \mathrm{C}$ e acima de $31^{\circ} \mathrm{C}$ não são favoráveis para a oviposição de $O$. insidiosus.

O aumento do número de ovos/dia/fêmea foi diretamente proporcional ao incremento da temperatura, na faixa de 16 a $25^{\circ} \mathrm{C}$, estabilizando-se nas temperaturas acima de $25^{\circ} \mathrm{C}$, com ajuste a uma curva de $2^{\circ}$ grau (Figura 4A), com médias de 5,6; 6,5 e 5,4 ovos/fềmea/dia, nas temperaturas de 25,28 e $31^{\circ} \mathrm{C}$, respectivamente. Resultado semelhante foi encontrado para $O$. insidiosus por Bush et al. (1993) com média de 6,8 ovos/fêmea/dia a $25^{\circ} \mathrm{C}$, quando as fềmeas foram alimentadas com ovos de Heliothis virescens (Fabricius) e superior aos de Argolo et al. (2002) quando esse predador foi alimentado com ovos de A. kuehniella a $25^{\circ} \mathrm{C}(3,4$ ovos/fềmea/dia).

O número total de ovos/fềmea de $O$. insidiosus foi também influenciado pela temperatura. Foi verificado um menor número de ovos/fêmea ( 35,4 ovos) quando as mesmas foram mantidas na temperatura de $16^{\circ} \mathrm{C}$. Os valores se ajustaram a uma curva de $2^{\circ}$ grau (Figura 4B), com aumento no número de ovos colocados pelas fêmeas, aliado ao aumento da temperatura, mas com estabilização nas temperaturas de 22 e $25^{\circ} \mathrm{C}(183,0$ e 206,7 ovos, respectivamente) e diminuição na temperatura mais alta $31^{\circ} \mathrm{C}$ (109,4 ovos).

Tommasini \& Nicoli (1993) encontraram uma média de 143 ovos/fêmea de $O$. insidiosus a $26^{\circ} \mathrm{C}$ e 16 horas de fotofase, quando as mesmas foram alimentadas com ovos de A. kuehniella. Argolo et al. (2002) verificaram 195 ovos/fêmea a $25^{\circ} \mathrm{C}$ e 12 horas de fotofase, sendo esse número semelhante ao observado no presente estudo. 
A influência da baixa temperatura $\left(16^{\circ} \mathrm{C}\right)$ na fecundidade de $O$. insidiosus foi evidente, ou seja, fêmeas desse predador nessa temperatura e em fotoperíodo 12L: 12E apresentaram comportamento de oviposição diferenciado em relação a demais temperaturas estudadas, com maior período de pré-oviposição (20,6 dias), menor porcentagem de fềmeas que ovipositaram $(60,0 \%)$ (Tabela 1) e menor número de ovos/fêmea $(35,4$ ovos) (Figura 2).

Adultos de $O$. insidiosus presentes em regiões temperadas, podem entrar em diapausa durante o inverno (Van Den Meiracker, 1999; Ruberson et al., 2000). Entretanto, essa condição é mais rapidamente reconhecida em fềmeas, as quais apresentam estruturas reprodutivas reduzidas e corpo gorduroso aumentado quando em diapausa (Ruberson \& Elkassabany, 1996). Já para espécies de regiões tropicais esse fenômeno ainda é desconhecido. Também segundo Beck (1980) o desempenho distinto de espécies presentes em regiões temperadas e tropicais pode estar relacionado à latitude e o fotoperíodo, de acordo com Van Den Meiraker (1999), é o fator primário para indução de diapausa reprodutiva em $O$. insidiosus em regiões temperadas. Assim, a baixa fecundidade de $O$. insidiosus a $16^{\circ} \mathrm{C}$ representa uma maior sensibilidade a essa temperatura e mais estudos são necessários quanto à diapausa reprodutiva desse predador presente em região tropical.

Com relação à curva média de oviposição de $O$. insidiosus, na temperatura de $25^{\circ} \mathrm{C}$ foi observado o pico de oviposição no $11^{\circ}$ dia da fase adulta (Figura 3), com média diária de ovos colocados de aproximadamente $5 \%$ do total de ovos colocados durante toda a sua vida. Até o $30^{\circ}$ dia após a emergência, as fêmeas colocaram $80 \%$ do total de ovos e, depois desse dia, o número de ovos diminuiram (Figura 3). Esses dados são essenciais para subsidiar aspectos da criação massal desse predador, podendo-se avaliar o 
período de coleta de ovos em laboratório e também a capacidade reprodutiva desse inseto no campo.

Quanto à longevidade de $O$. insidiosus sob diferentes temperaturas a que foram submetidos, observou-se que, tanto para machos como para fêmeas, houve um ajuste ao mesmo tipo de equação, ou seja, de $3^{\circ}$ grau (Figura 4). Para as fêmeas, ocorreu estabilização na longevidade em temperaturas acima de $28^{\circ} \mathrm{C}$, com menor longevidade verificada a 28 e $31^{\circ} \mathrm{C}$ ( 31,4 e 29,2 dias, respectivamente) (Figura 4A). Para machos, nas temperaturas de $16,22,25,28$ e $31^{\circ} \mathrm{C}$ foi observada diminuição gradativa na sua longevidade (Figura 4B).

Embora a temperatura não tenha ação isolada, o conhecimento da influência desse fator nos parâmetros reprodutivos de $O$. insidiosus é fundamental para subsidiar pesquisas com esse inseto no laboratório e no campo, e, determinar o seu potencial de multiplicação em condições de criações massal, parâmetros de controle de qualidade e também a sua efetividade em estudos de liberação e/ou introdução.

Tais estudos também fornecerão maior entendimento dos requerimentos e padrões sazonais desse predador, permitindo previsões mais acuradas das atividades e ocorrência das suas populações e, conseqüentemente, na sua atuação como agente de controle biológico de tripes em cultivos de regiões tropicais. O. insidiosus mostrou-se sensível quanto à sua reprodução e longevidade frente as diferentes temperaturas a que foi submetido, sendo $25^{\circ} \mathrm{C}$ a mais adequada para a multiplicação em laboratório. 


\section{CONCLUSÕES}

1. As diferentes temperaturas influenciam a reprodução e a longevidade de $O$. insidiosus.

2. Sob as temperaturas extremas $\left(16\right.$ e $\left.31^{\circ} \mathrm{C}\right)$, uma menor porcentagem de fêmeas oviposita.

3. O maior número de ovos é colocado a 22 e $25^{\circ} \mathrm{C}$.

4. O pico de oviposição a $25^{\circ} \mathrm{C}$ ocorre no $11^{\circ}$ dia e até o $30^{\circ}$ dia, após o período de pré-oviposição, as fêmeas colocam $80 \%$ dos ovos.

\section{AGRADECIMENTOS}

Ao Conselho Nacional de Desenvolvimento Científico e Tecnológico (CNPq) e Coordenação de Aperfeiçoamento de Pessoal de Nível Superior (CAPES) pelas bolsas de estudos e a FAPEMIG (Fundação e Apoio a Pesquisa do Estado de Minas Gerais) pelo apoio financeiro.

\section{REFERÊNCIAS BIBLIOGRÁFICAS}

ALAUZET, C., DARGAGNON, D.; MALUSA. J.C., 1994. Bionomics of a polyphagous predator: Orius laevigatus (Het. Anthocoridae). Entomophaga. 39: 33-40.

ARGOLO, V.M., BUENO, V.H.P.; SILVEIRA, L.C.P, 2002. Influência do fotoperiodo na reprodução e longevidade de Orius insidiosus (Say) (Heteroptera:Anthocoridae). Neotrop. Entomol. 31: 257 - 261.

BECK, S. D, 1980. Insect photoperiodism. New York, Academic Press, $387 p$.

BUENO, V.H.P, 2000. Desenvolvimento e multiplicação de percevejos predadores do gênero Orius Wolff. p. 69-90. In: BUENO, V.H.P. (ed.), Controle biológico de pragas: produção massal e controle de qualidade. Lavras, UFLA, 207p. 
BUSH, L., KRING T.J; RUBERSON, J.R, 1993. Suitability of greenbugs, cotton aphids, and Heliothis virescens eggs for the development and reproduction of Orius insidiosus. Entomol. Exp. Appl. 67: 217-222.

CAMPBEL, A.; FRAZER, B.D; GILBERT, N; GUITIERREZ, A.P; MACKAUER, M, 1974. Temperature requirements of some aphids and their parasites. J. Appl. Ecol. 11: 431-438.

CHYZIK, R., KLEIN, M; BENNDOV, Y, 1995. Overwintering biology of the predatory bug Orius albidipennis (Hemiptera: Anthocoridae) in Israel. Biocontrol Science and Tecnology 5: 287-296.

MALAIS, M.P; RAVENSBERG, W.J, 2003. The biology of glasshouse pest and their natural enemies: knowing and recognizing. Koppert, Netherlands, $109 \mathrm{p}$.

MENDES, S.M; BUENO, V.H.P, 2001. Biologia de Orius insidiosus (Say) (Hemiptera: Anthocoridae) alimentado com Caliothrips phaseoli (Hood) (Thysanoptera: Thripidae). Neotrop. Entomol. 30: 423-428.

MENDES, S.M.; BUENO, V.H.P; ARGOLO, V.M; SILVEIRA, L.C.P, 2002. Type of prey influences biology and consumption rate of Orius insidiosus (Hemiptera: Anthocoridae). Rev. Bras. Entomol. 46: 99-103. MENDES, S.M.; BUENO, V.H.P; CARVALHO, L.M; SILVEIRA, L.C.P, 2003. Efeito da densidade de ninfas de Aphis gossypii Glover, 1887 (Hemiptera: Anthocoridae) no consumo alimentar e aspectos biológicos de Orius insidiosus (Say, 1832) (Hemiptera: Anthocoridae). Rev. Bras. Entomol. 47: 19-24.

ROY, M.; BRODEUR, J; CLOUTIER, C, 2002. Relationship between temperature and developmental rate of Stetorus punctillum (Coleoptera: Coccinellidade) and its prey Tetranychus medalieli (Acarina: Tetranichidae). Biol. Contr. 31: 177-186. 
RUBERSON, J.R.; SHEN Y.J; KRING, T, 2000. Photoperiodic sensitivity and diapause in the predator Orius insidiosus (Heteroptera: Anthocoridae). Ann. Entomol. Soc. Amer. 93: 1123-1129.

SCOTT, A.J; KNOTT, M.A, 1974. A cluster analyses method for grouping means in the analyses of variance. Biometrics 30: 507-512.

SILVEIRA, L.C.P; BUENO, V.H.P, 2003. Orius insidiosus (Say, 1832) (Hemiptera: Anthocoridae): sensibilidade ao fotoperíodo e diapausa reprodutiva? Rev. Bras. Entomol. 47: 631-635.

TOMMASINI, M.G; NICOLI, G, 1993. Adult activity of four Orius species reared on two preys. IOBC/ WPRS Bull. 16: 281-284.

TOMMASINI, M.G; BENUZZI, D, 1996. Influence of temperature on the developments time and adult activity of Orius laevigatus. IOBC/ WPRS Bull. 19: 179-182.

TOMMASINI, M.G, 2003. Evaluation of Orius species for biological control of Frankliniella occidentalis (Pergande) (Thysanoptera: Thripidae). Wageningen University, Wageningen. Thesis (PhD). 214 p. VAN DEN MEIRACKER, R.A.F, 1994. Induction and termination for diapause in Orius predatory bugs. Entomol. Exp. Appl. 73: 127-137. VAN DEN MEIRACKER, R.A.F, 1999. Biocontrol of western flower thrips by heteropteran bugs. Amsterdan University, Amsterdam. Thesis (PhD). 145 p. 
Tabela 1. Porcentagem de fêmeas de $O$. insidiosus que ovipositaram em diferentes temperaturas, $70 \pm 10 \%$ de UR e fotofase de 12 horas.

Temperatura $\left({ }^{\circ} \mathrm{C}\right) \quad$ \% Fêmeas que ovipositaram

\begin{tabular}{lc}
\hline 16 & $60,0 \pm 11,24 \mathrm{~b}$ \\
19 & $81,8 \pm 12,19 \mathrm{a}$ \\
22 & $92,8 \pm 7,14 \mathrm{a}$ \\
25 & $100,0 \pm 0,00 \mathrm{a}$ \\
28 & $93,3 \pm 6,65 \mathrm{a}$ \\
31 & $73,68 \pm 10,38 \mathrm{~b}$ \\
\hline
\end{tabular}

${ }^{1}$ Médias seguidas de mesma letra, nas colunas, não diferem entre si pelo teste de Scott \& Knott $(\mathrm{P}<0,01)$. 

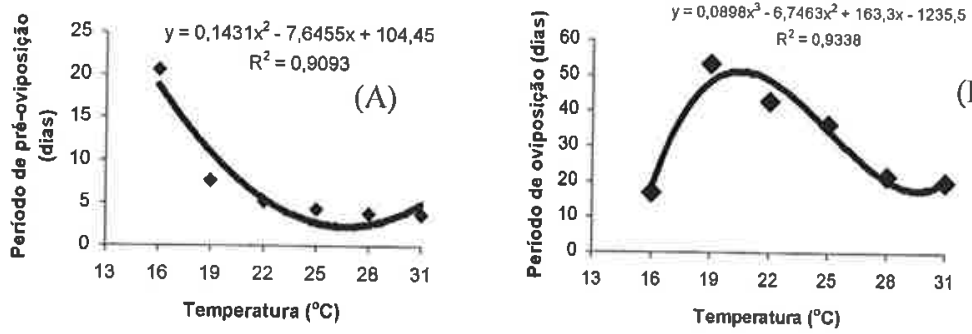

(B)

Figura 1. Períodos de pré-oviposição (A) e oviposição (B) de $O$, insidiosus em diferentes temperaturas, $70 \pm 10 \%$ de UR e fotofase de 12 horas.
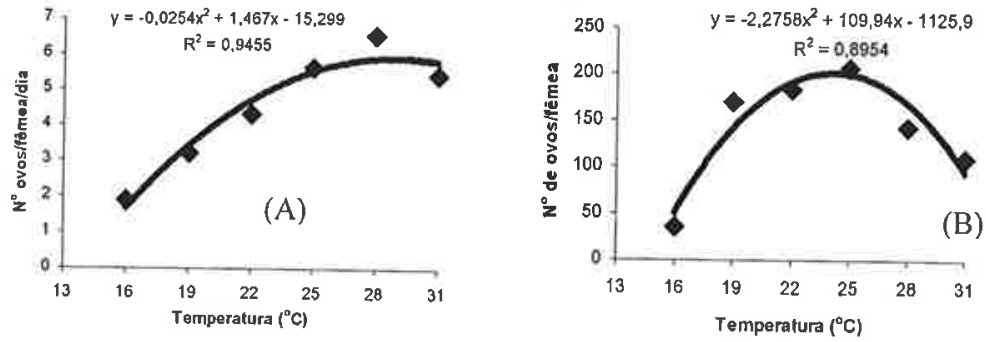

Figura 2. Número médio diário (A) e total (B) de ovos/fềmea de $O$. insidiosus em diferentes temperaturas, $70 \pm 10 \%$ de UR e fotofase de 12 horas. 
Figura 3. Curva de oviposição média de $O$. insidiosus a $25^{\circ} \mathrm{C}, 70 \pm 10 \%$ de

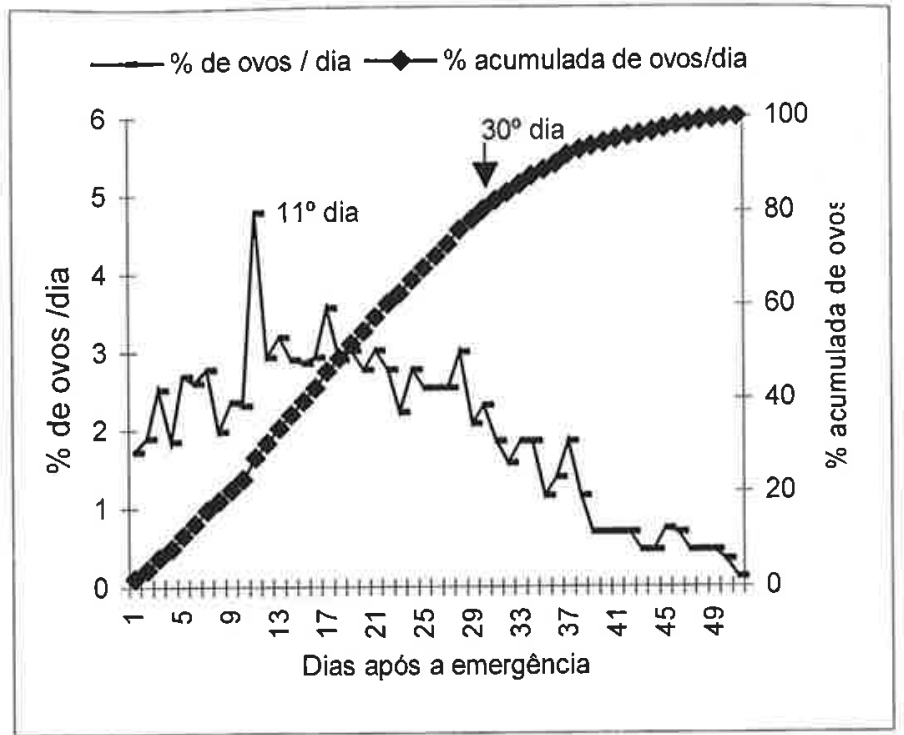

UR e fotofase de 12 horas.
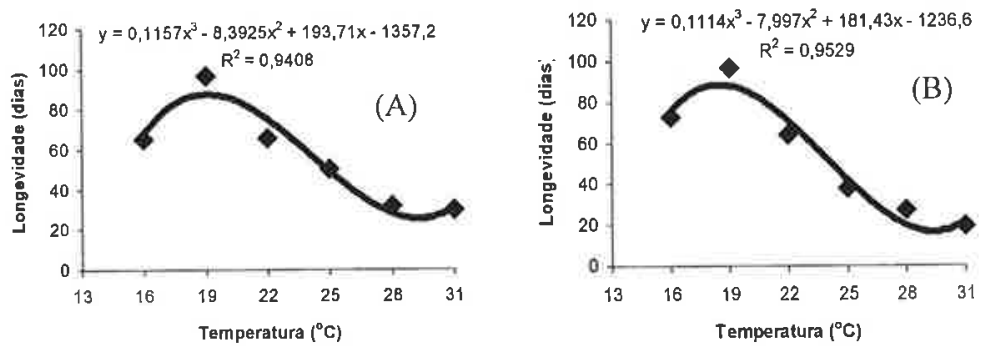

Figura 4. Longevidade de fềmea (A) e macho (B) de $O$. insidiosus sob diferentes temperaturas, $70 \pm 10 \%$ de UR e fotofase de 12 horas. 